\title{
El liderazgo transformacional y la motivación hacia el ámbito naval: una revisión conceptual
}

\section{Transformational leadership and motivation toward the naval field: a conceptual review}

\author{
Francisco Javier Chavez Freire \\ Universidad Naval, Ecuador \\ Luis Ángel Valle Lituma \\ Universidad de Especialidades Espíritu Santo, Ecuador
}

Autor para correspondencia: fchavezf@armada.mil.ec.

Fecha de recepción: 23 de junio de 2018 - Fecha de aceptación: 27 de agosto de 2018

Resumen: El presente trabajo aborda un análisis de la relación entre dos variables que son la motivación y el liderazgo transformacional, enmarcados en un ambiente militar con enfoque naval en el que las personas se desarrollan bajo diferentes situaciones de labor como patrullajes, navegaciones, entre otros, que, sumado a la rutina de la milicia y en muchos casos el riesgo que se corre, crea una disminución en el desempeño laboral, por lo cual es importante que el líder observe y genere acciones positivas enfocadas al desempeño de sus subordinados, de tal manera, se realizó una revisión teórica y se utilizaron métodos científicos de investigación tales como focus group y entrevistas dirigidas al personal de la Armada del Ecuador como sustento complementario de los conceptos y reflexiones que se exponen, llegando a una acertada relación entre las variables estudiadas.

Palabras clave: motivación; liderazgo transformacional; liderazgo militar y desempeño

\begin{abstract}
This paper deals with an analysis of the relationship between two variables that are motivation and transformational leadership, framed in a military environment with naval approach where people are developed under different work situations as patrols, sailing, etc. That joined the military routine and in many cases the risk involved, creates a decrease in work performance, which is important for the leader to observe and generate positive actions for the performance of their subordinates in command. It has been made with a theoretical revision and using scientific methods of investigation like focus groups and interviews to the personnel of the Ecuadorian Navy that strengthened the concepts and reflections that are exposed reaching a correct relation between the studied variables.
\end{abstract}

Key words: motivation; transformational leadership; military leadership and job performance 


\section{Introducción}

En el ámbito militar en Fuerzas Armadas existen muchas manifestaciones de liderazgo, que mucho dependen del individuo que comanda a un grupo determinado de personas, además estas pueden variar dependiendo de la actividad que se desarrolle o de la rama que involucre. Es muy probable que el tipo de liderazgo que se aplique dependa de la formación del individuo que se encuentra al mando y si este es del Ejército, la Fuerza Aérea o Armada (Marina).

Son diferentes actividades a la que el personal militar en el ámbito naval está sometido: operaciones militares en fronteras, navegaciones largas, patrullajes u operaciones de Aero vigilancia, etc. En ellas existe un riesgo que es asumido de una manera firme y profesional, lo que, sumado a la rutina, probablemente provocan que el desempeño laboral disminuya en el personal de tal manera que el líder debe ser muy observador, a fin de motivar a sus seguidores y adecuar su liderazgo de la mejor manera para el cumplimiento de la misión (Delbón, 2002).

La motivación es un factor importante, pero la influencia en el liderazgo transformacional desde un enfoque militar provoca el análisis de la relación existente entre ambos. De existir una relación cercana con el estilo de liderazgo mencionado, se obtendrá la pauta para la realización de acciones de motivación y ellos deben contribuir al mejoramiento del desempeño de los subordinados en las actividades que ejecuten por su profesión. El desempeño laboral abarca diversos factores, uno de ellos es la motivación, sin embargo, esta puede o no estar presente en los estilos de liderazgo como es el caso del liderazgo transformacional, y es necesario determinar la relación entre ambos en las organizaciones militares de orden Naval donde la motivación juega un papel importante.

El objetivo del presente trabajo es analizar la relación existente entre la motivación y el liderazgo transformacional aplicado al ámbito militar con un enfoque hacia el personal Naval y su desempeño. Se establecen definiciones, las principales características del liderazgo transformacional ligando su aplicación a la institución militar como lo es la Armada del Ecuador, finalmente revisando la motivación en el desempeño del personal utilizando el liderazgo transformacional y fortalecer la conceptualización mediante metodología cualitativa presentada a través de herramientas prácticas como la entrevista y focus group en donde se evidencia lo expresado en la teoría.

De acuerdo con el contenido mencionado anteriormente se estructuró el trabajo en dos partes que son la revisión teórica, abordando el liderazgo transformacional, el liderazgo naval y la motivación y por otro lado el análisis practico aplicando las herramientas cualitativas para el estudio central.

\section{Revisión Teórica}

Debido a la globalización y el constante cambio existente causado por la actividad de la humanidad en la que se presentan formas de guerra diferentes, con uso de moderna tecnología y las diversas misiones que emergen, provocan nuevas funciones en las Fuerzas Armadas, por lo que deben efectuarse cambios en la organización de carácter operacional, administrativo y 
orgánico. De esta manera, se genera la necesidad de que se replantee las habilidades y conocimientos que un líder requiere (Halpin, 2011; Laurence, 2011).

Las diferentes misiones con la que cumplen los militares, considerando las tradicionales como el combate o también llamado guerra, y las nuevas amenazas (guerra asimétrica) como misiones de paz, ayuda humanitaria, contraterrorismo, narcotráfico y piratería, los despliega durante largos periodos de tiempo a situaciones exigentes, estresantes y de riesgo (Yammarino, Mumford, Connelly, \& Dionne, 2010). Ante esta realidad las Fuerzas Armadas y en este caso la Armada del Ecuador necesitan de líderes que sean más innovadores para que puedan desenvolverse adaptándose al contexto de situaciones diversas e incertidumbre de sucesos (Pulakos, Arad, Donovan, \& Plamondon, 2000).

Según Morath, Leonard, \& Zaccaro (2011) la doctrina de liderazgo actual que lleva el ejercito de los Estados Unidos establece que un líder debe tener cualidades como creatividad, flexibilidad, adaptación, agilidad, versatilidad y motivación para formarse constantemente y entender los entornos cambiantes e inciertos, sin embargo, introducir innovación en este ámbito puede acarrear problemas ya que, en contenido de guerra, la equivocación puede conllevar a la muerte. Por tal motivo, en la actualidad los líderes deben entrenarse adquiriendo nuevas competencias para manejar la complejidad de los sistemas y crear diversas formas de control y consenso (Guiñazú, 2004), previniendo el sabotaje, observando marcos de referencia diferentes, con el objetivo de entender los obstáculos a los que se enfrenta en este caso la Armada y así poder innovar con el sistema de mando y estructura jerárquica propia (Castro \& Martina, 2003).

Halpin (2011) recomienda que el personal (Oficiales y tripulantes), deben capacitarse desde temprana edad en estas nuevas competencias, de tal manera que adquieran nuevas habilidades y no solo acaten órdenes de los superiores jerárquicos coartando sus capacidades. A pesar del entorno cambiante y del enfoque que se recomienda aplicar, ciertos componentes del liderazgo en la institución Naval se mantienen debido a que son importantes para la ejecución del mismo, entre estos la motivación, trabajo en equipo y la conducción de los subordinados, llamado también don de mando, además de la confianza que debe crearse como factor importante para influir en los seguidores (Sweeney, Thompson, \& Blanton, 2009).

\section{Liderazgo Naval.}

De acuerdo con Eberly, Johnson, Hernandez, \& Avolio (2013), el liderazgo es un proceso social de influencia que sucede no solo en las organizaciones sino en otros aspectos de la vida, por lo que el líder debe saber identificar en qué condiciones se produce una situación, para eso depende mucho del grado de madurez que posea (Hersey \& Blanchard, 1982). Entonces se debe integrar la relación que tiene el líder con sus subordinados y la conducta, de esta manera se evalúa el grado de madurez que posee considerando la experiencia, competencia, interés y motivación para realizar las tareas asignadas y adquirir responsabilidades (Sanchez , 2000).

En el transcurso de la historia el militar en grado de Oficial ejercía una autoridad rígida sobre su tripulación, a pesar de no obtener obediencia y lealtad siempre, sin embargo, en esta autoridad absoluta existía el respeto mutuo, como individuo y camarada, debido a las metas comunes, la misión y sobretodo la importancia de contar con cada hombre sin importar 
especialidad o rango (Gutierrez, 1996). Es entonces como el Almirante Nelson logra la cooperación de sus subalternos a través de la sólida confianza en sí y sus marinos, utilizando frases inspiradoras, gallardetes, palabras y demás acciones que motiven a su tripulación y la flota en general (Gutierrez, 2006).

Por lo anteriormente expuesto, el liderazgo militar está ligado a la motivación y a como los líderes pueden influir en sus seguidores (Godoy \& Bresó, 2013). Sin embargo, existen muchos estilos de liderazgo aplicables en la Organización Naval que pueden ser eficientes de acuerdo con las situaciones que se generen (Bardera, Gracía-Silgo, \& Pator , 2014). En la actualidad las organizaciones, incluyendo las militares, están sufriendo adaptaciones conforme a los sucesos del mundo moderno, de tal manera que necesitan cambios en sus políticas organizacionales, re direccionamiento de los recursos humanos, mejorar la gestión de personal y tener mayor liderazgo transformacional (Feinberg, Ostroff, \& Burke, 2005).

\section{Liderazgo transformacional en el ámbito Naval.}

Se menciona al liderazgo transformacional porque dentro del contexto laboral es un elemento crítico con la capacidad de influir en la positividad de como los equipos y personas aprecian su trabajo (Christian, Garza, \& Slaughter, 2011). Es así como este estilo de liderazgo se ha ido desarrollando y tomando fuerza por medio de la investigación en el transcurso de los últimos años (Mendoza , Ortiz, \& Parker, 2007), convirtiéndose en uno de los estilos para estudio más analizados. El liderazgo transformacional surge por medio de los trabajos realizados por Burns(1978) y House(1971) acerca del liderazgo carismático, el cual se considera como un proceso relacional entre el líder y sus seguidores con efectos bidireccionales entre estos.

Cruz, Salanova, \& Martínez(2013) mencionan que en la actualidad hablar de liderazgo no solo se centra en estudiar al líder como figura individual sino también el entorno, la cultura y sobre todo a los seguidores, premisa que se confirma por medio de los estudios de Avolio(2007), en los que determina que el liderazgo es una dinámica compartida y compleja de carácter social. Es por ello que Bass(1985) define el liderazgo transformacional claramente como un proceso motivacional de los seguidores o en este caso de los subordinados en el que el líder transforma sus valores, actitudes y rendimiento. También debe crear una conexión en la que pueda relacionarse con otros individuos elevando así la moral y motivación de sus seguidores (Espinar \& Jose, 2012), de tal manera que el líder pueda responder a sus necesidades y brindar ayuda para desarrollar al máximo sus potenciales (Northouse, 2001).

De igual forma Bono \& Judge(2002) demostraron la influencia positiva del liderazgo transformacional en el desempeño y compromiso obteniendo personal motivado y satisfecho siendo identificado este liderazgo como un agente de cambio para los líderes y seguidores. Así mismo, Hetland \& Sandal(2003) ratificaron que existe una significativa influencia en la motivación por arriba de otros estilos de liderazgo, con ello dando soporte a la teoría que indica que mencionada influencia es lo que hace exitoso y único al liderazgo transformacional (Bass, 2008).

El líder militar posee una formación progresiva y permanente durante su carrera, enfocados en tres aspectos que son la conducción, comando y mando (Delbón, 2002), que se establecen en los diferentes grados, desde ser Jefe de Sistemas de Armas de un buque en el grado 
de Alférez, luego comandante de ese buque en el grado de Capitán hasta ser Comandante de Escuadra $^{1}$ en grado de Almirante. Además, el ejercicio del mando está presente en todo momento, lo que hace que el líder cultive su personalidad militar e interiorice sus procedimientos y principios de mando generando características como experiencia, conocimiento y calidad de liderazgo lo cual es reconocido por sus subalternos o subordinados (Olid, 2002).

El liderazgo transformacional describe a un líder como a una persona capaz de inspirar a sus seguidores, aumentando su madurez y motivación, logrando que vayan más allá de lo requerido, buscando la excelencia y calidad, es decir, lograr tener una visión compartida líderseguidor (Cruz, Salanova, \& Martínez, 2011), una característica del líder militar que se contrasta con la determinación de poder formar una cultura y ambiente para que se produzca un cambio organizacional (Koontz \& Heinz, 2003).

Hablar de liderazgo también es observar el ambiente laboral que está estrechamente ligado al bienestar en donde la Psicología del Trabajo y las Organizaciones toma parte a fin de considerar estos factores como una meta propuesta para la organización (Salanova, 2008). De igual manera Sparr \& Sonnentag(2008) relacionaron positivamente el liderazgo transformacional con el bienestar de los seguidores, por lo que influye directamente en el desempeño laboral. Para analizar el desempeño laboral del militar se toma en cuenta ciertos factores externos como la cohesión grupal, trabajo en equipo y clima positivo, que en el contexto militar son de relevancia para la formación y enseñanza de los mandos militares (García, 2013).Como factores internos que favorecen positivamente al liderazgo están la autoconciencia, personalidad, aceptación y autocontrol (Bartone, 2006).Con estos factores expuestos, el liderazgo transformacional ha demostrado que los líderes promueven enfoques positivos de las situaciones de estrés transformándolas en desafíos (Lyons \& Schneider, 2009).

Schultz, Greenley, \& Brown(1995) también afirman que el liderazgo trasformacional favorece a la coherencia de trabajos, claridad de metas y satisfacción laboral, de manera que influyen en la creencia e interpretación de los subordinados con respecto a su trabajo, incrementando el bienestar, reduciendo el estrés y contribuyendo a una labor proactiva a favor de la satisfacción (Ugoani, Amu, \& kalu, 2015).

\section{La motivación en el personal Naval.}

Según Gutierrez (2015) la Motivación es una variable de personalidad que influye en el deseo de los individuos de satisfacer los requisitos establecidos para un rol en una organización, siendo el caso de la Armada y su personal que la conforma, custodiar la soberanía de los espacios acuáticos y mar territorial. Conociendo plenamente esto, el personal Naval se desarrolla en la profesión de las armas con una visión clara de estar preparados para el combate, en donde la motivación juega un papel muy importante, ya que es una preparación mental para una acción de conflicto o guerra (Mc Gregor, 1975). Entonces es importante que los esfuerzos en entrenamiento sean extremos para simular las condiciones de combate real y enfrentar momentos de tensión máxima, esfuerzo físico y psicológico (Torres, 2012).

Es necesario fomentar y alentar actividades que promuevan el trabajo en equipo y estimulen la motivación a través del entrenamiento y régimen diario (Aguirre \& Guevara, 2012)

\footnotetext{
${ }^{1}$ Grupo determinado de buques de guerra de un mismo modelo o diferente pertenecientes a la Armada.
} 
de tal manera que se estrechen los lazos interpersonales generando mayor compromiso de cada integrante para cumplir con su tarea (Stouffer, 1949).

Se debe permanentemente confiar en nuestro personal y lograr lo más difícil, que ellos confíen en sus mandos (Phillips \& Lord, 1981). La base para ello radica en una buena comunicación, la disposición de "tener siempre abierta la puerta de la oficina" o incluso solamente los oídos y la habilidad de poder retransmitir, en un lenguaje claro y sencillo, el propósito de una determinada tarea, jamás subestimar su comprensión o esperar sólo respuestas mecánicas (Kolditz, Millen, \& Wong, 2014). También se debe recordar que los ejemplos se establecen por medio del contacto diario entre los subordinados y que de las experiencias navales queda un legado de aquellos que se retiran, creando una fuente de tradiciones en el liderazgo de la Armada, ya que esta, establece demandas únicas en condiciones de alto riesgo en donde la fe en el líder, su gente y su causa deben ser incólumes (Bauer, 1986).

Para aseverar lo conceptualizado en esta revisión teórica y considerando que no existe un análisis anterior que se enfoque en la Armada del Ecuador, se investigaron de manera cualitativa las variables establecidas, primero por medio de un focus group o reunión de grupo, técnica muy exitosa, debido a que de las personas seleccionadas representan un segmento de la población, permitiendo obtener una gran cantidad de ideas, puntos de vista distintos e interacción en un corto periodo de tiempo sobre un tema seleccionado (Martínez, 2008). Así también, combina la dimensión social y psicológica con capacidad de diagnóstico alcanzando un nivel de profundidad en la discusión de los participantes (Shaw, 1995).

Como segunda técnica, la entrevista presenta la posibilidad de ahondar en el punto de vista de una persona representante de un segmento de realidad social, quien puede conjugar el lenguaje, emociones y acciones con base a un tema propuesto (Rogers, 1961). Según Robles (2011) la entrevista permite el discurso espontáneo y libre de la persona entrevistada sin que el moderador condicione sus intervenciones.

\section{Análisis De Los Resultados De Los Métodos De Investigación}

\section{Liderazgo aplicado en la Armada del Ecuador.}

Una vez realizada la revisión teórica en cuanto al liderazgo transformacional y la motivación enfocados al ámbito naval con un detalle conceptualizado a través de diferentes textos y autores, es necesario fortalecer estos conceptos por medio de la aplicación de métodos cualitativos de investigación, con cuyos resultados se evidenciará la aplicación de estos conceptos en el diario vivir de algunas personas que conforman la Armada del Ecuador.

En el proceso se aplicarán dos métodos:

- Focus group

- $\quad$ Entrevistas

El focus group fue orientado a oficiales subalternos, comprendidos en el grado de alférez de fragata, teniente de fragata y teniente de navío. Líderes de personal naval en escalafones inferiores que generalmente se desempeñan a nivel departamental u operacional, entre estos, 
oficiales en las especialidades de Superficie, Infantería de Marina, Guardacostas, Submarinistas, Abastecimientos, de género masculino y femenino en un total de diez personas.

Según la Guía de Pauta que consta en los anexos, se realizó la sesión abarcando temas puntuales como el liderazgo y su aplicación, el conocimiento acerca del liderazgo transformacional, factores que puedan incidir en su aplicación y la motivación como en el desarrollo de un liderazgo efectivo para el cumplimiento de objetivos. Basado en el marco de la revisión teórica y de la relación entre estas dos variables, se realizaron diferentes preguntas, entre las más importantes: ¿Cómo aplicas el liderazgo?, ¿Qué factores consideran importantes en el liderazgo?, ¿Cómo influye la motivación en el desempeño de una persona?, ¿Qué observas en la Armada acerca del Liderazgo Transformacional?

En la discusión de acuerdo a los puntos planteados se obtuvieron significativas conclusiones que fortalecen el planteamiento conceptual, que son las siguientes:

- Si bien es cierto que el liderazgo transformacional se asemeja a un liderazgo carismático, esto no significa que el líder debe plantearse con una actitud totalmente empática y benevolente ya que perdería el enfoque principal de conducir a sus subordinados al cumplimiento del objetivo.

- Es fundamental que el líder genere un buen ambiente de trabajo y camaradería con la finalidad de obtener el cumplimiento cabal de las disposiciones que emita, observando en los subordinados la buena predisposición para el efecto con una certeza de que las ordenes serán cumplidas y de forma proactiva, mas no solo por cumplir la tarea encomendada.

- La motivación es importante al momento de ejercer liderazgo, sin embargo, para poder motivar se debe saber llegar a los subordinados a través del tacto, el tino y otras cualidades más que son necesarias en un líder, entonces la motivación es un factor principal indudablemente, pero se deben tener en cuenta estas otras cualidades que integradas llevarán al resultado deseado.

- El liderazgo no solo se ejerce en niveles de mando, sino en cualquier grado jerarquizado y es deber del líder no solo fortalecer las habilidades que tiene un subordinado, sino generar otras que ayuden a su mejoramiento personal y a su vez su adaptabilidad a incursionar en cualquier ámbito que se le disponga con una buena motivación. Así también se debe formar nuevas generaciones de líderes que en ausencia del principal tengan la capacidad de reaccionar y conducir al grupo de acuerdo con las situaciones que se presenten.

Las entrevistas estuvieron direccionadas a oficiales superiores en el grado de Capitán de Fragata y Capitán de Corbeta de género masculino y femenino respectivamente, en las edades de cuarenta a cincuenta años, que por su jerarquía se encuentra a un nivel de pensamiento estratégico comandando Unidades o Repartos Navales.

Se estableció una guía de pauta para la ejecución de las entrevistas de acuerdo a como se detalla en el anexo, conteniendo tópicos como los tipos de liderazgo, el liderazgo y la motivación, resultados de la motivación en las personas, y con el planteamiento de preguntas. Fueron seleccionados el Capitán de Fragata Boris Rodas Cornejo y la señorita Capitán de 
Corbeta Alexandra Chica Cedeño. En el caso del Sr. Capitán de Fragata, este ha comandado unidades a flote de la Armada con un total de veinte y siete años de servicio y quince años de embarque efectivo, además de estar al mando de Unidades educativas y administrativas, lo cual le da una amplia experiencia en la conducción de personas que se desarrollan en diferentes entornos ya mencionados anteriormente.

Por otro lado, la Srta. Capitán de Corbeta posee especialidades en administración y educación desenvolviéndose como directora de Institutos Académicos de la Armada con una experiencia de 20 años de servicio y actualmente comandando el Centro Tecnológico Naval, centro que otorga los títulos de nivel tecnológico a los tripulantes de la Armada

De acuerdo a las entrevistas realizadas se puede contrastar y fortalecer la conceptualización, evidenciando ciertos puntos que se mencionan a continuación:

- Para ejercer un liderazgo efectivo es importante relacionarse con las personas, no solo en el ámbito laboral, sino también en lo personal sin llegar a inmiscuirse directamente en los asuntos personales. Sin embargo, es necesario conocer a fondo a la persona, tener un acercamiento, amistades, problemas que puedan tener, para demostrar el interés que se tiene como líder en el lado humano del grupo de personas que se tiene bajo el mando, con esto genera apertura y confiabilidad para el manejo de conflictos o situaciones laborales que tal vez disminuyan la eficiencia laboral. De esta manera se pueden abordar directamente los problemas, potencializar las habilidades de las personas creando la sensación de utilidad en su trabajo, identidad y aporte considerable para el logro de objetivos comunes.

- Esta relación que existe entre los subordinados y el líder permite una comunicación asertiva, favoreciendo a la motivación en la organización y el sentido de pertenencia, caso contrario el comportamiento de las personas cambia, disminuyendo la productividad, la predisposición e ingresando a un estado de confort demostrando un trabajo mecánico o automático.

- El ejemplo es un factor de motivación y relación entre los subordinados, por lo cual el líder debe propender la participación en su organización y evidentemente demostrar calidad moral o espiritual, caballerosidad y otras cualidades más que lo visualicen como un modelo a seguir.

- Es necesario conocer al grupo de trabajo al cual se va a liderar y también el entorno en que se desempeñan, ya que no todos los repartos navales son iguales o tienen las mismas características, esto dará las primeras pautas para saber qué tipo de liderazgo utilizar, pues puede suceder casos en el que se necesitará un liderazgo autoritario y tradicional de la milicia hasta obtener los cambios requeridos en la personas para ejercer un liderazgo transformacional, generando así, un camino para que el líder pueda resaltar las cualidades de sus subordinados y su importancia para la Institución, en otras palabras "motivar", con esto se consigue comprometimiento en el rol asignado a cada uno de ellos.

Con lo expuesto, indudablemente las ideas vertidas en el focus group y las entrevistas a los señores oficiales de grado superior fortalecen y confirman los enfoques teóricos revisados.

\section{Conclusiones}


El liderazgo se evidencia en el personal Naval desde muy temprana edad, empezando por las escuelas de formación en donde se enseña acerca del don de mando y la conducción de personas, es allí donde empiezan a experimentar el ejercicio del liderazgo y emergen las habilidades de líder. Esto no significa que una vez finalizada su etapa de formación termina su liderazgo, sino todo lo contrario, porque a lo largo de su carrera desde los rangos inferiores hasta los superiores, el entorno y el mismo ambiente de trabajo de una organización jerarquizada y militar, provocarán que el líder gane experiencia, madurez y confianza en el campo, logrando desenvolverse de una correcta manera generando acciones pertinentes a cada situación que se le presente.

El grado de madurez que posee el líder Naval está asociado directamente a la motivación, es decir, se observará cuan motivador puede ser y esto también dependerá de las actividades que ha realizado durante su carrera como patrullajes, misiones operacionales o navegaciones, en las que simplemente la ejecución de estas sumado al contacto y relación que tiene con los subordinados es inminente y permanente. Como ejemplo al realizar un navegación en un buque de guerra los subordinados se ven sometidos a guardias de control, pocas horas de descanso, talvez reparaciones de maquinaria, etc, que provocan una rutina agotadora y dependiendo del tiempo que dure esto, el desempeño del personal puede disminuir, por lo que con un liderazgo transformacional, el líder puede persuadir positivamente y lograr un cambio en la forma de pensar de sus subordinados, observando que la agotadora rutina puede significar abnegación hacia la labor que estan realizando con un objetivo común y la obtención de la satisfacción del deber cumplido.

El liderazgo transformacional se enfoca en grupos o equipos de trabajo, de tal manera que es vital la labor conjunta que realizan los subordinados en cada una de sus áreas correspondientes para que un buque pueda navegar y todos sus sistemas estén en correcto funcionamiento, ya que el mínimo error puede generar riesgo no solo a uno sino a toda la dotación, es por eso que el trabajo que desempeña cada uno, desde el menos antiguo hasta el comandante tienen el mismo grado de importancia. Así mismo, el liderazgo transformacional analiza las situaciones y el entorno que se desarrolla, al igual que el líder Naval, esto significa que también observa y considera el bienestar de los subordinados y el nivel de satisfacción que ellos poseen, que en el caso de no ser los adecuados influirá en el desempeño de los mismos.

Cuando se trata temas relacionados al ámbito militar, son muy escasos los estudios o trabajos que se encuentra, sobre todo a nivel de Sudamérica, y no solo respecto a liderazgo y motivación, sino a un sin número de temas más, que Fuerzas Armadas, como parte de la sociedad, forman parte de un eje de desarrollo y principalmente de la defensa de las naciones. En el transcurrir del tiempo la profesión Naval se torna más compleja. Se desarrollan nuevos sistemas de armas, producto de nuevos descubrimientos científicos, generando nuevas capacidades y con ello el empleo de nuevas tácticas, conceptos estratégicos y empleo del Poder Naval, de tal manera que el personal naval debe poseer una adaptación rápida al cambio, flexibilidad de pensamiento e imaginación para la aplicación de nuevas ideas en mejora de las capacidades navales y de la Institución. 
En vista de esto, es recomendable continuar con estudios con base al liderazgo, tomando en cuenta que el entorno actual en el que se desempeñan Fuerzas Armadas es cambiante y requiere de adaptación por parte de sus miembros. La búsqueda de un estilo de liderazgo efectivo podría ser una herramienta fundamental para el desarrollo de las Instituciones militares y de igual manera para los comandantes en la toma de decisiones estratégicas para la conducción de personas. También la mujer toma parte de las organizaciones modernas y su aporte es tan importante como el del hombre en las mismas capacidades y equidad, por lo que es recomendable analizar el liderazgo de género en la milicia y su desarrollo en el transcurrir del tiempo.

Finalmente, en el ámbito Naval, el uso práctico de la motivación y el liderazgo transformacional son la forma de afrontar positivamente las situaciones del entorno moderno para el cumplimiento de los objetivos institucionales y la misión encomendada, enmarcados en valores, principios, con mística y decisión para el beneficio y porvenir del país.

\section{Bibliografía}

Aguirre, E., \& Guevara, G. (2012). Factores asociados con el desempeño del personal que labora como oficiales de transito y seguridad de la Universidad de Costa Rica. Revenf, 1-10.

Avolio, B. (2007). Promoting more integrative strategies for leadership theory building. American Psychologist, 25-33.

Bardera, M., Gracía-Silgo, M., \& Pator , A. (2014). Gestión de estrés en la Fuerzas Armadas. Revista del Instituto Español de Estudios Estratégicos (IEEE), 1-24.

Bartone, P. (2006). Resilience under military operational stress: can leaders influence hardiness? Military Psychology, 18.

Bauer, L. (1986). "Return to tradition". United States Institute Proceedings, 9-32.

Bass, B. (1985). Leadership and Performance Beyond Expectations. New york: The Free Press.

Bass, B. (2008). Handbook of Leadership: Theory, Research, and Application. Free Press.

Bono, J., \& Judge, T. (2002). Self-concordance at work: Toward understanding the motivational effects of transformational leaders. Academy of Management Journal, 554-572.

Burns, J. (1978). Leadership. Harper and Row.

Castro, A., \& Martina, M. (2003). Concepciones de civiles y militares argentinos sobre el liderazgo. Boletín de Psicología, 63-79.

Christian, M., Garza, A., \& Slaughter, J. (2011). Work engagement: A quantitative review and test of its relations with task and contextual performance. Personnel Psychology, 89-136. 
Cruz, V., Salanova, M., \& Martínez, I. (2011). Liderazgo Transformacional y su relación con el capital psicológico positivo grupal. Sesión de cartel presentado en la III jornada de emociones y bienestar. Madrid.

Cruz, V., Salanova, M., \& Martínez, I. (2013). Liderazgo transformacional: investigación actual y retos futuros. Universidad y Empresa, 13-32.

Delbón, A. (2002). La formación de líderes militares. Segundo encuentro de reflexión y experiencias sobre liderazgo efectivo. Ponencia: Instituto Universitario Naval.

Eberly, M., Johnson, M., Hernandez, M., \& Avolio, B. (2013). An integrative process model of leadership: examining loci, mechanisms, and event cycles. American Psychologist, 427443.

Espinar, R., \& Jose, O. (2012). Motivation: The Road to Successful Learning. Profile, 17, 125136.

Feinberg, B., Ostroff, C., \& Burke, W. (2005). The role of within-group agreement in understanding transformational leadership". Journal of Occupational and Organizational Psychology, 471-488.

García, M. (2013). Revisión de programas de resiliencia basados en la evidencia de los ejércitos. Sanidad Militar.

Godoy, R., \& Bresó, E. (2013). ¿Es el liderazgo transformacional determinante en la motivación intrínseca de los seguidores? Journal of Work and Organizational Psychology, 59-64.

Guiñazú, G. (2004). Capacitación efectiva en la empresa. INVENIO, 104-114.

Gutierrez , O. (2015). Estudios de Liderazgo y Género. Revismar, 34-39.

Gutierrez, O. (1996). El Liderazgo Militar. Revismar, 113(832), 3.

Gutierrez, O. (2006). Literatura sobre Liderazgo Naval. Revismar, 252-255.

Halpin, S. (2011). Historical influences on the changing nature of leadership within the military environment. Military Psychology, 23, 479-488.

Hersey, P., \& Blanchard, K. (1982). Management of organizational behavior: utilizing human resources. Prenticehall.

Hetland, H., \& Sandal, G. (2003). Transformational leadership in Norway: Outcomes and personality correlates. European Journal of Work and Organizational Psychology, 147170. 
House, R. (1971). A path goal theory of leader effectiveness. Administrative Science Quarterly, 321-338.

Kolditz, T. A., Millen, R. A., \& Wong, L. (2014). Why They Fight: Combat Motivation in the Iraq War. Pennsylvania: Paperback.

Koontz, H., \& Heinz, W. (2003). Administración: Una perspectiva global. Mexico: Mcgraw Hill.

Laurence, J. (2011). Military Leadership and thee Complexity of combat and culture. Military Psychology, 489-501.

Lyons, J., \& Schneider, T. (2009). Leadership and stress: The effects of leadership style on stress outcomes. Leadership Quarterly(20).

Mc Gregor, D. (1975). Leadership and Motivation. Milán: M.I.T press.

Mendoza , I., Ortiz, M., \& Parker, H. (2007). Dos décadas de investigación y desarrollo en liderazgo transformacional. Revista del Centro de Educación, 25-41.

Morath, R., Leonard, A., \& Zaccaro, S. (2011). Military leadership: an overview and introduction to the special issue. Military Psychology, 453-461.

Northouse, P. (2001). Leadership Theory and Practice. Thousand Oaks,CA.: Sage Publications, Inc.

Olid, P. (2002). Liderazgo Militar. Military Review, 20-29.

Phillips, J., \& Lord, R. (1981). Causal attributions and perceptions of leadership. Leader-ship Quaterly, 203-231.

Pulakos, E., Arad, S., Donovan, M., \& Plamondon, K. (2000). Adaptability in the workplace: development of a taxonomy of adaptive performance. Journal of Applied Psychology, 612624.

Robles , B. (Sep. de 2011). SciELO. Recuperado el 5 de 7 de 2017, de http://www.scielo.org.mx/scielo.php?script=sci_arttext\&pid=S018516592011000300004

Rogers, C. (1961). El proceso de covertirse en persona. Editorial Paidós.

Salanova, M. (2008). Organizaciones saludables: Una aproximación desde la Psicología Positiva. Psicología Positiva Aplicada, 403-427.

Sanchez , E. (2000). Teoría del liderazgo situacional en la administración local:validez del modelo. Psicothema, 435-439. 
Shaw, M. (1995). "Dinámica de grupo, Psicolgía de la conducta de los pequeños grupos". Editorial Herder.

Schultz, R., Greenley, J., \& Brown, R. (1995). Organization. Management and client effects on staff burnout. Journal of Health and Social Behavior(36).

Sparr, J., \& Sonnentag, S. (2008). Fairness perceptions of supervisor feedback, LMX, and employee well-being at work. European Journal of Work and Organizational Psychology, 198-225.

Stouffer, S. (1949). The American Soldier. New Jersey: Princeton University Press.

Sweeney, P., Thompson, V., \& Blanton, H. (2009). Trust and influence in combat: an interdependence model. Journal of Applied Social Psychology, 235-264.

Torres, O. (2012). Motivación para el combate. REVISMAR, 159-166.

Ugoani, J., Amu, C., \& kalu, E. (2015). Dimensions of emotional intelligence and Transformational leadership: A correlation analysis. Independent Journal of Management \& Production (IJM\&P), 563-584.

Yammarino, F., Mumford, M., Connelly, M., \& Dionne, S. (2010). Leadership and team dynamics for dangerous military contexts. Military Psychology. Military Psychology, 15-41. 